Artículo

\title{
Antagonismo de Trichoderma spp. vs hongos asociados a la marchitez de chile
}

\author{
Petra Andrade-Hoyos ${ }^{1}$ \\ Alfonso Luna-Cruz ${ }^{2 \S}$ \\ Eduardo Osorio-Hernández ${ }^{3}$ \\ Eduardo Molina-Gayosso ${ }^{4}$ \\ Nadia Landero-Valenzuela ${ }^{5}$ \\ Hebert Jair Barrales-Cureño ${ }^{1}$
}

\begin{abstract}
${ }^{1}$ División de Procesos Naturales-Universidad Intercultural del Estado de Puebla. Calle principal a Lipuntahuaca s/n, Lipuntahuaca, Huehuetla, Puebla. CP. 73475. (andrahoy@gmail.com; hebert.jair@uiep.edu.mx). ${ }^{2}$ CONACYT-Instituto de Investigaciones Químico Biológicas-Universidad Michoacana de San Nicolás de Hidalgo. Av. Francisco J. Mújica s/n, Ciudad Universitaria, Morelia, Michoacán. CP. 58030. ํDivisión de Estudios de Posgrado-Universidad Autónoma de Tamaulipas. Matamoros s/n, Zona Centro, Ciudad Victoria, Tamaulipas. CP. 87000. (eosorio@ docentes.uat.edu.mx). ${ }^{4}$ Ingeniería en Biotecnología-Universidad Politécnica de Puebla. Tercer carril del ejido Serrano s/n, San Mateo Cuanalá, Juan C. Bonilla, Puebla. CP. 72640. (eduardo.molina@uppuebla.edu.mx). ${ }^{5}$ Universidad Politécnica Francisco I. Madero-Ingeniería en Agrotecnología. Tepatepec s/n, Francisco I. Madero, Hidalgo. CP.42660. (nlanderova@ conacyt.mx).
\end{abstract}

§Autor para correspondencia: alunacr@conacyt.mx.

\section{Resumen}

En México se siembran más de 100 variedades de chile (Capsicum annuum L.), es un cultivo altamente redituable y representa una actividad económica de importancia nacional. Actualmente, enfrenta graves problemas fitosanitarios por la presencia de enfermedades como la marchitez del chile, causada por un complejo de patógenos de suelo. Para el control de estas enfermedades, se utilizan fungicidas tóxicos y residuales, que contaminan el ambiente e inducen resistencia genética en los fitopatógenos. Por ello, es necesario buscar alternativas de control para solucionar este problema, por tanto, el objetivo de este trabajo fue evaluar el efecto antagónico de Trichoderma spp., contra los agentes causales de marchitez en confrontaciones in vitro mediante cultivos duales. El estudio se llevó acabo en 2016, en la Universidad Intercultural de Estado de Puebla, Huehuetla, Puebla, México. Se evaluaron tres aislamientos de Trichoderma provenientes de la rizósferas de árboles de aguacate, $T$. viride, T. harzianum y T. asperellum y tres aislamientos de los fitopatógenos $P$. capsici, $F$. oxysporum y $R$. solani obtenidos de las raíces de plantas de chile con síntomas de la marchitez. Los aislamientos se caracterizaron morfológicamente para su identificación. Los tres hongos antagonistas presentaron alto porcentaje de inhibición, T. asperellum mostró el 88.25\%, $T$. viride $87.22 \%$ y $T$. harzianum con $87.8 \%$. En las condiciones del estudio, T. asperellum, T. viride y $T$. harzianum, resultaron microorganismos antagónicos eficientes contra los fitopatógenos. Se sugiere realizar evaluaciones de biocontrol con estos aislamientos en invernadero y a campo abierto.

\section{Palabras clave: Capsicun annum, Phytophthora capsici, Trichoderma spp.}

Recibido: mayo de 2019

Aceptado: agosto de 2019 


\section{Introducción}

El cultivo de chile (Capsicum annuum L.) en México, enfrenta graves problemas fitosanitarios que limitan su producción a causa de enfermedades como la marchitez del chile, inducida por un complejo de fitopatógenos de suelo, entre ellos Phytophthora capsici Leo (Hernández-Castillo et al., 2014), Rhizoctonia solani Kühn y Fusarium oxysporum (Vásquez et al., 2009). Los principales síntomas que provoca esta enfermedad son la muerte prematura de las plantas al causar la obstrucción y desequilibrio del funcionamiento de los haces vasculares, defoliación, cambios de color, rizado del follaje, daño a estructuras reproductivas, maduración adelantada e irregular, pudrición de la raíz, necrosis del tallo y ocurrencia de rebrotes (Granke et al., 2012, Rivera et al., 2018).

Para controlar la incidencia y severidad de estos patógenos, se utilizan fungicidas altamente tóxicos y residuales que provocan un impacto ambiental negativo, contaminando el suelo, el aire y los mantos acuíferos, además intoxican al ser humano, crean resistencia genética en los fitopatógenos a los ingredientes activos (Matar et al., 2009; Osorio et al., 2016; Hernández-Hernández et al., 2018) e incrementan los costos de producción. Otra consecuencia es la reducción del área de siembra debido a la infestación del suelo es el aumento en la severidad e incidencia de la enfermedad y reducción de los rendimientos obtenidos por superficie sembrada (García, 2010).

La relevancia del género Trichoderma radica en la eficiencia que posee para combatir enfermedades, se ha reportado en múltiples estudios el impacto que tiene sobre las pudriciones de raíz, problemática que se encuentra entre las enfermedades que causan pérdidas económicas significativas en diferentes cultivos agrícolas. Aunque los productos químicos son aún la principal herramienta de control para estas enfermedades, los agentes biológicos son una manera efectiva para proporcionar un control más rápido y más seguro (Verma et al., 2007).

Se ha investigado durante más de 70 años los mecanismos de control biológico de las especies de Trichoderma spp. (Martínez et al., 2013), su capacidad antagónica está ampliamente documentada como agentes de biocontrol de diversos patógenos que afectan cultivos de importancia agrícola y económica (Infante et al., 2009; Mayo et al., 2015). Las cepas de Trichoderma producen enzimas extracelulares (Osorio-Hernández et al., 2016), compiten con los hongos patógenos por espacio y nutrientes al reducir o detener completamente el desarrollo del fitopatógeno (Sánchez-García et al., 2017), promueven el crecimiento de las plantas e inducen resistencia sistémica, micoparasitismo y antibiosis mediante acción restrictiva directa a los hongos fitopatógenos.

Estos mecanismos se ven favorecidos por la habilidad de los aislamientos de Trichoderma para colonizar la rizósfera de las plantas. (Leandro et al., 2007; Woo y Lorito, 2007; Guédez et al., 2012). Las diferentes especies de Trichoderma son una alternativa para el biocontrol contra diversos fitopatógenos de suelo (Singh et al., 2011) entre ellos P. capsici, F. oxysporum y R. solani, además de estimular el incremento de la biomasa radicular y el área foliar (Ezziyyani et al., 2005; Ezziyyani et al., 2007). Por lo anterior, el objetivo de la presente investigación fue determinar el efecto antagónico de Trichoderma spp., como método de biocontrol para el complejo de fitopatógenos que causan marchitez del chile en evaluaciones in vitro mediante cultivos duales. 


\section{Materiales y métodos}

\section{Ubicación del estudio}

El presente trabajo se llevó a cabo en el Laboratorio de Biología de la Universidad Intercultural del Estado de Puebla, Huehuetla, Puebla, México. Sus coordenadas geográficas son los paralelos $20^{\circ}$ 01 ' $48^{\prime \prime}$ y $20^{\circ} 09^{\prime} 12^{\prime \prime}$ de latitud norte y los meridianos $97^{\circ} 35^{\prime} 00^{\prime \prime}$ y $97^{\circ} 40^{\prime} 24^{\prime \prime}$ de longitud oeste.

\section{Material fúngico}

Se aislaron de suelo tres especies de Trichoderma en medio papa dextrosa-agar (PDA). Se dispersó $1 \mathrm{~g}$ de suelo húmedo en cajas con medio PDA y se incubaron a $25 \pm 2{ }^{\circ} \mathrm{C}$ en oscuridad durante dos días, después se retiraron de la incubadora y se mantuvieron bajo lámpara de luz blanca por dos días para inducir esporulación de Trichoderma sp., del desarrollo de Trichoderma, se tomó una porción para transferir a medio de cultivo PDA e incubar a $25 \pm 2{ }^{\circ} \mathrm{C}$, esto se realizó hasta obtener crecimientos axénicos y puros, libres de otros hongos o bacterias. Después de la última esporulación en este medio, se conservaron en agua estéril y aceite mineral.

Los patógenos Rhizoctonia sp., Fusarium sp. y Phytophthora capsici se aislaron de raíces de chile, las raíces se lavaron con agua corriente; posteriormente, la porción de tejido se desinfestó por inmersión durante 1 min en solución 1.5\% de hipoclorito de sodio y se enjuagó con agua destilada estéril, las raíces se escurrieron sobre sanitas estériles, finalmente se sembraron fragmentos de 1 cm en medio PDA para el aislamiento y purificación de Rhizoctonia sp. y Fusarium sp.

En el caso del oomicete Phytophthora capsici se sembraron raíces de chile de $1.5 \mathrm{~cm}$ de longitud en medio agar-agar ${ }^{\circledR}$ con jugo- $\mathrm{V}^{\circledR}$ (jugo de 8 verduras) y se adicionaron antibióticos (Pimaricina $10 \mu \mathrm{g} \mathrm{L}{ }^{-1}$, Ampicilina $292 \mu \mathrm{g} \mathrm{L}{ }^{-1}$, Rifampicina $10 \mu \mathrm{g} \mathrm{L}{ }^{-1}$, Pentacloronitrobenceno $0.1 \mathrm{~g} \mathrm{~L}^{-1} \mathrm{e}$ Himexazol 0-25 $\mu \mathrm{g} \mathrm{L}^{-1}$ ), posteriormente se incubaron a $28 \pm 2{ }^{\circ} \mathrm{C}$ durante tres días (Andrade et al., 2012), previamente purificados los patógenos de la raíz del chile se mantuvieron bajo conservación en agua estéril con el fin de preservación (Molina-Gayosso et al., 2016). Previó a la confrontación dual los microorganismos se activaron en medio de cultivo PDA más ácido láctico (Figura 1).

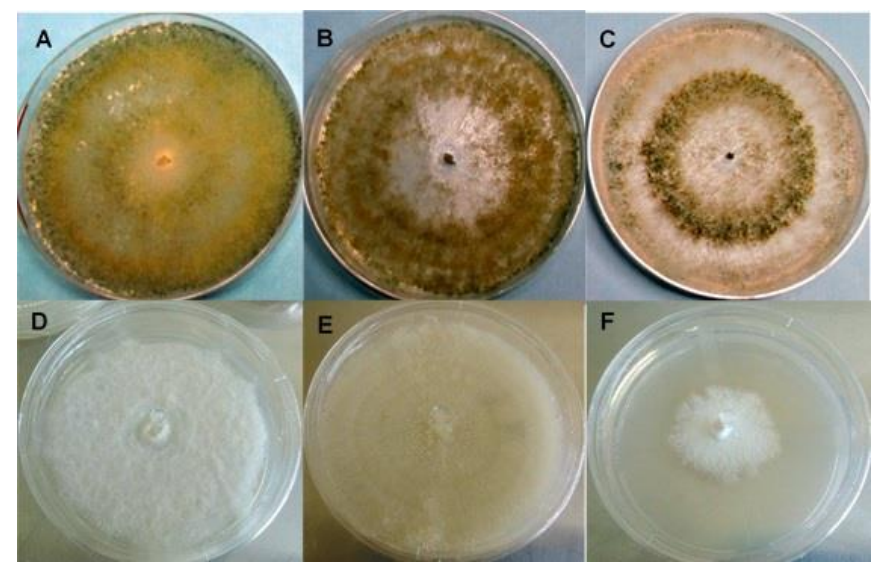

Figura 1. Crecimiento en medio de cultivo PDA de antagonistas y patógenos de la marchitez del chile. $\mathrm{A}=T$. viride $; \mathrm{B}=T$. harzianum $; \mathrm{C}=T$. asperellum $(\mathrm{C} 2) ; \mathrm{D}=$ Rhizoctonia $\mathrm{sp} . ; \mathrm{E}=P$. capcisi $; \mathrm{y}$ $\mathrm{F}=$ Fusarium $\mathbf{s p}$. 


\section{Caracterización morfológica}

Para estudiar las características morfológicas, tanto los aislamientos de los patógenos como de Trichoderma, se cultivaron en medio PDA y se incubaron a $24 \pm 2{ }^{\circ} \mathrm{C}$ durante $12 \mathrm{~h}$ bajo luz blanca (Rivera-Jiménez et al., 2018). En la identificación del género Fusarium sp. se utilizó como referencia la descripción de Burgess et al. (1994) y la identificación de la especie se realizó mediante las claves de Seifert (1996). La identificación de la especie de Rhizoctonia se realizó con base en Sneh et al. (1991) y la del oomicete Phytophthora sp. se caracterizó mediante las claves de Erwin y Ribeiro (1996); de Gallegly y Hong (2008).

Por último, en la caracterización morfológica de los aislamientos de Trichoderma spp. se utilizaron las claves taxonómicas de Barnett y Hunter (1972). Para los aislamientos de Trichoderma se utilizaron cultivos monoconidiales en combinación con la técnica de cámara húmeda (Harris, 1986). Un disco de PDA de $5 \mathrm{~mm}$ de diámetro se inoculó con la espora de Trichoderma y se colocó

entre un porta y cubreobjetos y esta preparación sobre un triángulo de varilla estéril dentro de una caja petri y se incubó a $25 \pm 2{ }^{\circ} \mathrm{C}$ durante $24 \mathrm{~h}$.

Las características macro y microscópicas a considerar fueron textura de la colonia, presencia de micelio, anillos concéntricos, tinción de la colonia al reverso, forma y tamaño de conidios y fiálides (Chaverri et al., 2015; Jang et al., 2018; Nawaz et al., 2018; Du Plessis et al., 2018), se observaron las estructuras de los aislamientos de Trichoderma spp. a 40X en un microscopio óptico (Zeiss Axiosiop plus).

\section{Tasa de crecimiento}

En la determinación de la tasa de crecimiento de micelio en antagonistas y patógenos, crecidos en PDA, se realizaron mediciones cada ocho horas.

\section{Actividad antagónica de Trichoderma sobre patógenos}

La técnica de cultivos duales se empleó para determinar la actividad antagónica de los diferentes aislamientos de Trichoderma sobre Rhizoctonia sp., Fusarium sp. y P. capsici (Sonnenbichler et $a l ., 1983$ ). Se empleó un diseño completamente aleatorizado (con dos factores: Trichoderma y los patógenos), con ocho repeticiones.

Los ensayos se realizaron evaluando cultivos duales de Trichoderma spp., contrapuestos con tres fitopatógenos, tres aislamientos de Trichoderma contra Rhizoctonia sp., Fusarium sp. y P. capsici. En la prueba de cultivos duales se utilizaron cajas petri con PDA libre de antibióticos, se depositó en un extremo un disco de $5 \mathrm{~mm}$ de diámetro con micelio activo del fitopatógeno (previamente crecido en PDA por cinco días) y en el extremo equidistante se colocó un disco de micelio (previamente crecido en PDA por cinco días) de $5 \mathrm{~mm}$ de diámetro con Trichoderma spp., las cajas se incubaron a $28 \pm 2{ }^{\circ} \mathrm{C}$ y se observaron cada $8 \mathrm{~h}$ para registrar el número de días al primer contacto entre el antagonista y el fitopatógeno, se midió el crecimiento de ambas colonias (cm) y se evaluó el porcentaje de inhibición del crecimiento micelial radial, basándose en la fórmula propuesta por Ezziyyani et al. (2007), calculando de la siguiente manera: 


$$
\mathrm{PICR} \%=\frac{\mathrm{R} 1-\mathrm{R} 2}{\mathrm{R} 1} * 100
$$

Donde: $\mathrm{PICR}=$ porcentaje de inhibición de crecimiento radial; $\mathrm{R} 1=$ crecimiento radial $(\mathrm{mm})$ del patógeno sin Trichoderma spp., $\mathrm{R} 2=$ crecimiento radial $(\mathrm{mm})$ del patógeno con Trichoderma spp.

\section{Micoparasitismo de Trichoderma sobre patógenos}

Para conocer la presencia de parasitismo de las cepas de Trichoderma hacia Rizoctonia sp., Fusarium sp. y P. capsici se empleó la técnica de microcultivos de Riddel descrita por Paul (1999). Esta técnica consiste en colocar dentro de una caja de petri una varilla de vidrio en forma de $\mathrm{V}$ y sobre ella, un portaobjetos estéril, sobre el cual se dispuso un disco de PDA de $10 \mathrm{~mm}$ de diámetro. La disposición de cada hongo se realizó en dos puntos cardinales, después de colocar el micelio de ambos hongos sobre el PDA, con una diferencia de $24 \mathrm{~h}$, primero se colocaron los patógenos con ayuda de una aguja estéril, los cuales tienen un crecimiento lento comparado con Trichoderma y se colocó un cubreobjetos estéril, se incubó a $25 \pm 2{ }^{\circ} \mathrm{C}$.

Cuando el disco de agar fue cubierto con el crecimiento de ambos hongos, el portaobjetos fue retirado de la caja de petri para preparar los frotis. El cubreobjetos fue colocado en otro portaobjeto limpio y provisto de una gota de colorante conocido como azul de algodón. Por otro lado, se retiró el disco de agar del portaobjetos original, se colocó una gota del colorante y un cubreobjetos. Una vez eliminado el exceso de colorante, las preparaciones fueron selladas y evaluadas microscópicamente, es decir se observaron 10 muestras para determinar si hubo o no parasitismo por las cepas de Trichoderma sp., se observó a 40X en un microscopio óptico (Zeiss Axiosiop plus).

\section{Análisis estadístico}

Los datos del PICR, expresados en porcentaje, se transformaron con el angular arcoseno $\sqrt{\mathrm{x}}+1$ (Steel et al., 1986) y en el crecimiento micelial en la prueba de biocontrol, se utilizó un diseño completamente aleatorizado (tres cepas de Trichoderma sp. y tres fitopatógenos) con ocho repeticiones. Los datos obtenidos para cada uno de los ensayos se sometieron a un análisis de varianza y una prueba de comparación de medias Tukey $(p \leq 0.05)$. Dichos datos se analizaron con el paquete estadístico SAS versión 9.0 para Windows (SAS, 2002).

\section{Resultados y discusión}

\section{Caracterización morfológica}

Los patógenos causantes de la marchitez del chile correspondieron con lo reportado por RiveraJiménez et al. (2018). Se encontró similitud con F. oxysporum, las colonias de crecimiento en PDA tenían abundante micelio aéreo y de color rosa pálido a morado intenso a color magenta con una textura algodonosa. Los macro-conidios presentaron de uno a cinco septos, los conidios midieron 23-54 × 3-4.5 $\mu \mathrm{m}$, respectivamente. En la identificación de Rhizoctonia solani correspondió con lo reportado por Sneh et al. (1991) y Lozano et al. (2015), R. solani se caracterizó por formar micelio aéreo, color marrón, en medio de cultivo PDA formo micelio hialino. 
Las hifas generalmente mostraron un ángulo recto y después de este se formó un septo, los diámetros de las hifas presentaron de 4.1 a $8.9 \mu \mathrm{m}$, lo que corresponde con Barnett y Hunter (1998). El aislamiento de Phytophthora capsici se caracterizó por formar esporangios ovoides, elongados, elipsoides con una o dos papilas, el tamaño de los esporangios fue de 35.8 a 64.7 x 19.6 a $25.2 \mu \mathrm{m}$., finalmente e grosor apical fue de 1.85 a $4.3 \mu \mathrm{m}$ (Erwin y Ribeiro, 1996; Gallegly y Hong, 2008).

De acuerdo con los criterios de identificación taxonómica de Barnett y Hunter (1972), las especies de Trichoderma se compararon con claves taxonómicas e identificadas por sus características morfológicas. Las especies de $T$. harzianum presentaron colonias color verde claro a oscuro con textura polvosa a algodonosa, con micelio, conidióforo y conidios de aspecto de color verde (Figura 2, Cuadro 1) muestran diversas ramificaciones en forma piramidal, en algunos casos se observa la formación de dos a tres ramas laterales y presenta micelio aéreo. En T. asperellum y T. viride se aprecia una coloración ausente al reverso de la placa petri.

Cuadro 1. Caracterización morfológica de Trichoderma spp.

\begin{tabular}{|c|c|c|c|c|c|c|c|c|c|}
\hline $\begin{array}{c}\text { Trichoderma } \\
\text { spp. }\end{array}$ & $\begin{array}{l}\text { Color de } \\
\text { colonia }\end{array}$ & $\begin{array}{c}\text { Textura de } \\
\text { colonia }\end{array}$ & $\begin{array}{l}\text { Micelio } \\
\text { aéreo }\end{array}$ & Anillos & $\begin{array}{l}\text { Color al } \\
\text { reverso }\end{array}$ & $\begin{array}{c}\text { Forma de } \\
\text { conidios }\end{array}$ & $\begin{array}{c}\text { Tamaño } \\
\text { conidios } \\
(\mu \mathrm{m})\end{array}$ & $\begin{array}{l}\text { Forma } \\
\text { fiálides }\end{array}$ & $\begin{array}{c}\text { Tamaño } \\
\text { fiálides } \\
(\mu \mathrm{m})\end{array}$ \\
\hline T. harzianum & $\begin{array}{c}\text { Verde } \\
\text { oscuro a } \\
\text { color } \\
\text { grisáceo }\end{array}$ & Algodono & Abundante & $\begin{array}{c}\text { Sin } \\
\text { presencia }\end{array}$ & & Subgloboso & $\begin{array}{l}3.8-4 \times \\
3.1-3.7\end{array}$ & Globosa & $\begin{array}{r}6.3-15.6 \\
\times 2.7-3.4\end{array}$ \\
\hline $\begin{array}{c}T . \\
\text { asperrellum }\end{array}$ & $\begin{array}{l}\text { verde } \\
\text { claro a } \\
\text { verde } \\
\text { oscuro }\end{array}$ & Polvoso & $\begin{array}{c}\text { Sin } \\
\text { presencia }\end{array}$ & $\begin{array}{l}\text { de } 3 \text { a } 4 \\
\text { anillos }\end{array}$ & Incoloro & $\begin{array}{l}\text { Ovoide a } \\
\text { elisoipde }\end{array}$ & $\begin{array}{c}4-4.4 x \\
3-3.5\end{array}$ & $\begin{array}{l}\text { Ligeramente } \\
\text { globoso }\end{array}$ & $\begin{array}{c}2.3-2.8 \\
\times 10.2- \\
11.8\end{array}$ \\
\hline T. viride & $\begin{array}{l}\text { Verde } \\
\text { oscuro }\end{array}$ & Algodonosa & Abundante & $\begin{array}{c}\text { Sin } \\
\text { presencia }\end{array}$ & Incoloro & $\begin{array}{l}\text { Globoso } \\
\text { Elipsoidal }\end{array}$ & $\begin{array}{c}4-4.8 \\
\times 3.5-4\end{array}$ & Lageniformes & $\begin{array}{c}8-14 \\
\times 2.4-3\end{array}$ \\
\hline
\end{tabular}

Las esporas y fiálides, estructuras características corresponden a especies de Trichoderma, presentaron formas y tañamos característicos de las especies de T. harzianum, T. asperellum y $T$. viride correspondiente a lo reportado por diferentes autores (Barnett et al., 1972; de Hoog, 2000; Romero-Arenas et al., 2009; Chaverri et al., 2015; Jaklitsch et al., 2015).
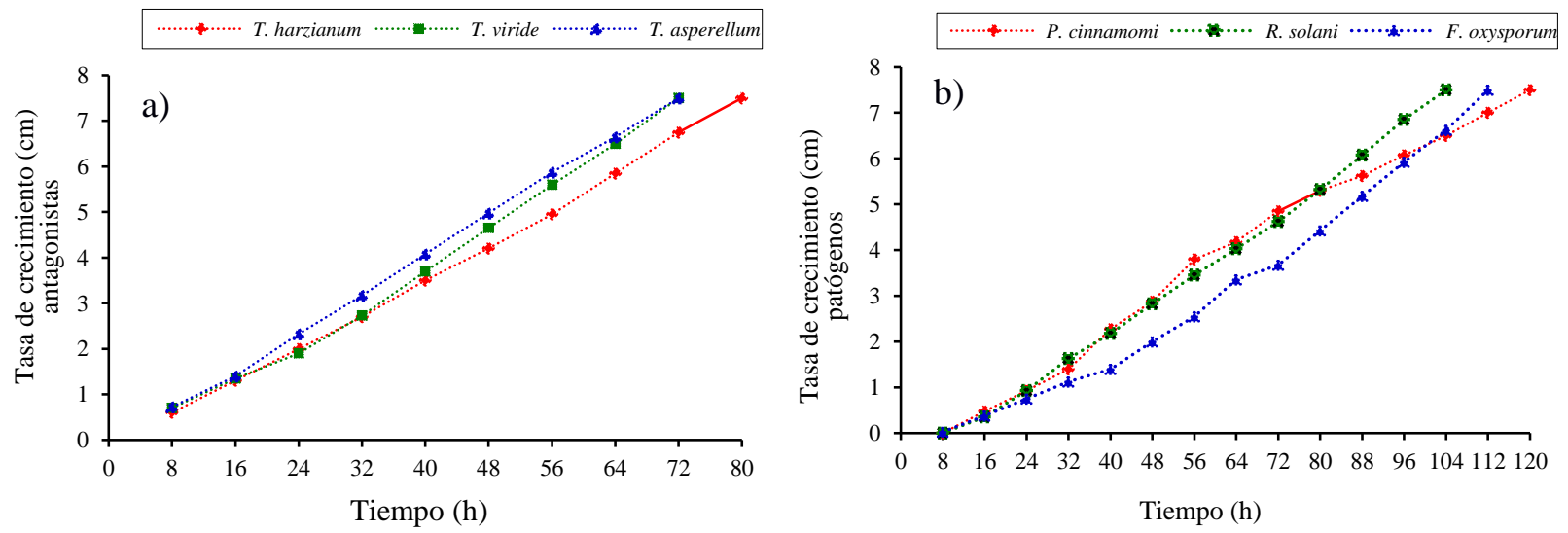

Figura 2. Tasa de crecimiento, a) $T$. harzianum, $T$. viride y $T$. asperellum; y b) $P$. capsici, $R$. solani y $F$. oxysporum. 


\section{Tasa de crecimiento}

La evaluación de la tasa de crecimiento inicial de los antagonistas y patógenos resultaron estar completamente relacionadas al iniciar crecimiento del micelio a las $8 \mathrm{~h}$; sin embargo, las tres especies de Trichoderma tienen la capacidad de antagonismo por completar el llenado de la caja petri en $80 \mathrm{~h}$. Estos resultados indica la importancia de conocer la velocidad de crecimiento de los patógenos, así como de los antagonistas de T. harzianum, T. viride y T. asperellum.

Los aislamientos de Trichoderma spp., mostraron un crecimiento más rápido que los patógenos, este comportamiento es promisorio en el control de patógenos de la raíz, además permitió este ensayo permitió considerarlo para las pruebas de PICR y dar ventaja de dos días a Fusarium oxysporum, Rhizoctonia solani y Phytophthora capsici.

\section{Actividad antagónica de Trichoderma sobre patógenos}

La actividad antagónica de $T$. viride contra $P$. capsici mostró diferencia significativa $(p \leq 0.05)$, debido a que $T$. viride presentó $0.516 \mathrm{~mm}$ en comparación de $P$. capsici que obtuvo $0.241 \mathrm{~mm}$ de crecimiento en caja petri con medio de cultivo; es decir, $T$. viride puede utilizarse como agente de control biológico, por ser eficaz contra $P$. capsici, ya que también se observó hiperparasitismo por parte del patógeno (Figura 3). Lo anterior, concuerda con lo reportado por Bouziane et al. (2016), al evaluar a $T$. viride contra $P$. infestans en pruebas in vitro, encontraron que el hongo benéfico mostró capacidad inhibitoria entre 58 y $68 \%$, resultados similares fueron al evaluar en plantas.

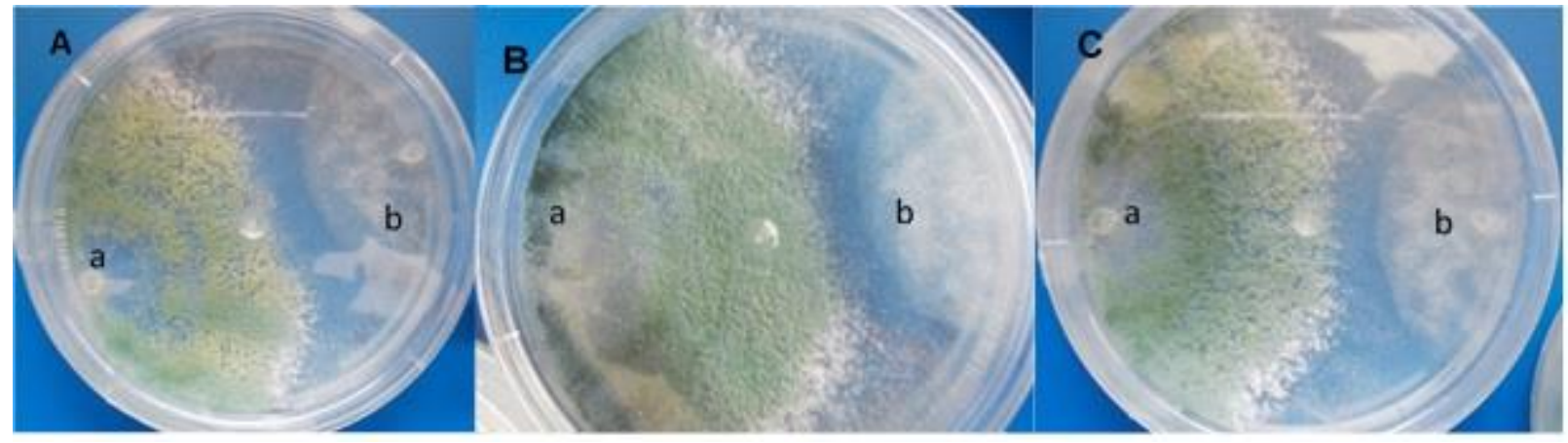

Figura 3. Crecimiento dual, confrontamientos in vitro con Trichoderma en contra de los patógenos, A) Trichoderma viride vs $P$. capsici; B) Trichoderma asperellum vs Fusarium oxysporum; y C) Trichoderma harzianum vs Rhizoctonia solani.

De igual forma, Zegeye et al. (2011), encontraron que $T$. viride mostró una inhibición completa en el crecimiento radial de $P$. infestans en pruebas in vitro. Además, mencionaron que la aplicación foliar de $T$. viride tiene un buen potencial para controlar a $P$. infestans en condiciones de invernadero. Por otra parte, la evaluación de antagonismo dual de $T$. viride contra $F$. oxysporum presentaron crecimiento de $0.656 \mathrm{~mm}$ del antagonista y $0.221 \mathrm{~mm}$ por parte del patógeno, esto evidencia como $T$. viride es apto para la utilización en el control de $F$. oxysporum.

En la confrontación dual $T$. viride contra $R$. solani obtuvieron un crecimiento del antagonista de $0.746 \mathrm{~mm}$ y del patógeno $0.112 \mathrm{~mm}$, los resultados de crecimiento por parte del antagonista fueron mayor en comparación con el patógeno (Figura 3). En ambos casos se han encontrado resultados 
similares donde los dos hongos son micoparasitados o destruidos por T. viride (Figura 3) ya que tiene un efecto antifúngico con este patógeno (Jhon et al., 2010; Perveen, 2012; Sánchez-García et al., 2017).

Es importante mencionar, que en las primeras $8 \mathrm{~h}$ el crecimiento de los antagonistas mostraron un crecimiento acelerado que propicia el encuentro con el patógeno en menor tiempo, indicativo que el antagonista creció o cubrió más superficie en cultivo in vitro.

Resultados similares mencionaron diversos autores donde demostraron que Trichoderma spp y $T$. viride son utilizados para el control biológico de $R$. solani (Sánchez-García et al., 2017). Se observó un efecto antifúngico, en el que inhibe el crecimiento y causa lisis a este patógeno. En las confrontaciones de $T$. viride contra los agentes causantes de la marchitez del chile. La evaluación del antagonismo de T. harzianum contra $P$. capsici, F. oxysporum y $R$. solani (Figura 4) presentó mayor crecimiento por parte del antagonista en comparación con el patógeno, denotando hiperparasitismo al invadir completamente al patógeno, resultado similar obtuvo OsorioHernández et al. (2011) en el que observó que Tricoderma spp., es un antagonista eficaz para el control de $P$. capsici, inhibiendo este patógeno hasta $48 \%$.
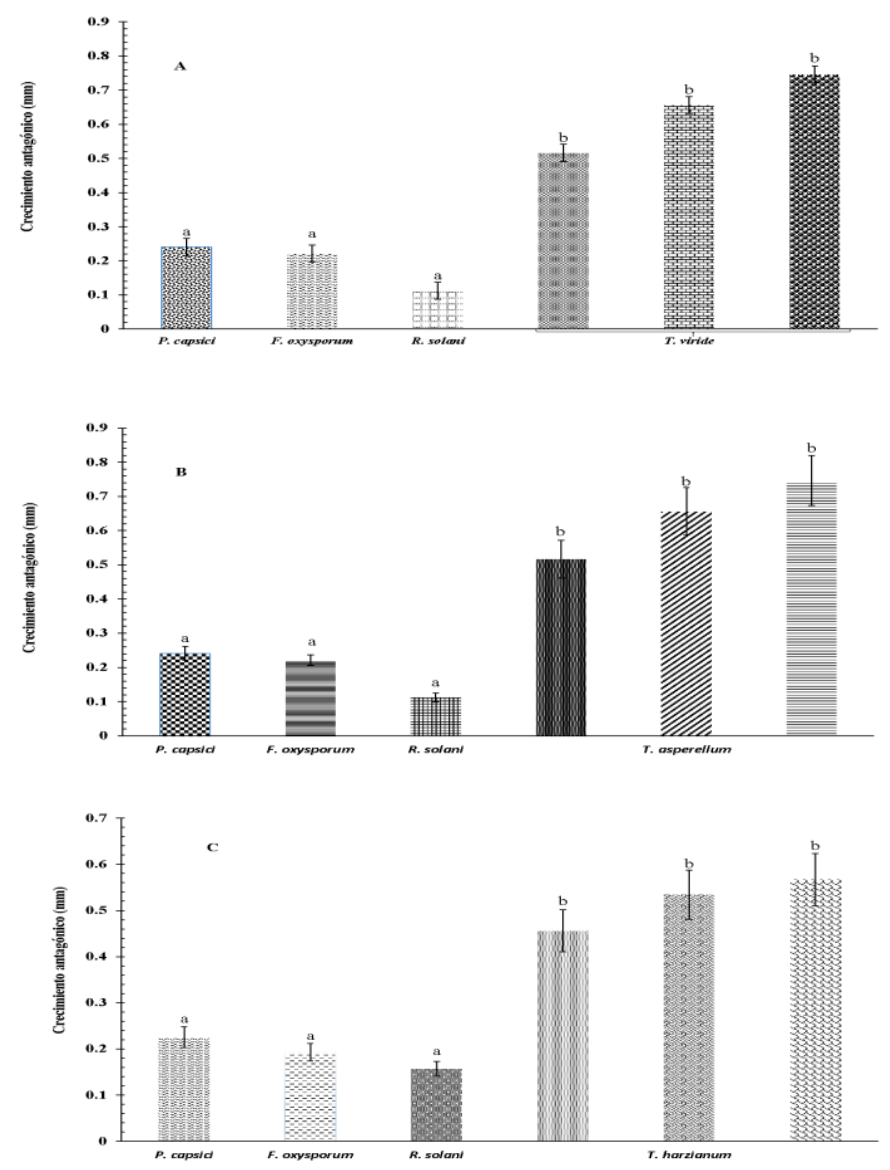

Figura 4. Crecimiento micelial (mm) en la prueba de biocontrol de A) T. viride vs $P$. capsici, $F$. oxysporum y $R$. solani; B) $T$. asperellum. Vs $P$. capsici, $F$. oxysporum y $R$. solani; y $C) T$. harzianum vs $P$. capsici, F. oxysporum y $R$. solani. 
Asimismo, en la confrontación de $T$. viride en contra de $F$. oxysporum y $R$. solani, se encontró la misma tendencia. Se encontró un valor significativo $(p \leq 0.05)$ en la evaluación de susceptibilidad con base al PICR de los tres patógenos responsables de la marchitez del chile; sin embargo, la mayor susceptibilidad la presentó Fusarium oxysporum con $92.68 \%$, seguido de Rizoctonia solani y $P$. capsici (Cuadro 2), resultados que prueban la eficacia de los antagonistas (Osorio et al., 2016) ante patógenos que afectan la raíz del chile desde plántulas y plantas en producción (Romero et al., 2017).

En la valoración del porcentaje de inhibición de crecimiento radial (PICR) de los patógenos causantes de la marchitez del chile no hubo diferencias significativas entre los antagonistas $T$. asperellum, $T$. viride y $T$. harzianum; por lo que se sugiere utilizar como agente de control biológico cualquiera de los tres Trichoderma; es decir, son efectivo por inhibir más de $85 \%$ a los patógenos causantes de la marchitez del chile (Cuadro 2), la respuesta de inhibición del crecimiento de fitopatógenos es debido a la síntesis de metabolitos secundarios y a los diferentes mecanismos de acción de micoparasitismo (Harman, 2006; Infante, 2009).

El comportamiento similar de inhibición de los tres tratamientos son favorables resultados enontrados por quienes reportan que por arriba de $80 \%$ de inhibición es aceptable en el biocontrol de patógenos que se transmiten por el suelo y por la eficacia de combatir enfermedades de la raíz (Hermosa et al., 2012; Sabbagh et al., 2017).

Cuadro 2. Evaluación de la susceptibilidad de los patógenos ante Trichoderma y porcentaje de inhibición de Trichoderma sp. contra los patógenos causantes de la marchitez del chile.

\begin{tabular}{cccc}
\hline Patógenos & $\begin{array}{c}\text { Susceptibilidad de patógenos ante } \\
\text { Trichoderma PICR }(\%)\end{array}$ & Trichoderma & $\begin{array}{c}\text { Actividad antagónica } \\
\text { Trichoderma PICR (\%) }\end{array}$ \\
\hline Fusarium oxysporum & $92.68 \mathrm{a}$ & T. asperellum & $88.25 \mathrm{a}$ \\
Rhizoctonia solani & $87.86 \mathrm{~b}$ & T. viride & $87.22 \mathrm{a}$ \\
Phytophthora capsici & $83.33 \mathrm{c}$ & T. harzianum & $87.8 \mathrm{a}$ \\
\hline
\end{tabular}

$\mathrm{a}, \mathrm{b}, \mathrm{c}=$ denota los tratamientos medios que son significativamente iguales o diferentes según la prueba de Tukey en $p<0.05$. PICR $=$ porcentaje de inhibición de crecimiento radial .

Los resultados de micoparasitismo de los antagonistas con frecuencia tuvo un crecimiento más favorable, cabe mencionar que las primeras $8 \mathrm{~h}$ fueron significativas $(p \leq 0.05)$ mostrando que el antagonista tuvo un crecimiento acelerado que propicia que el encuentro se pueda tener en un tiempo corto. Asimismo, se pudo observar que no solo presentó la inhibición del crecimiento de estos patógenos, sino que también presentó una invasión (hiperparasitismo) total del antagonista hacia el patógeno inclusive esporulando sobre el patógeno. En un estudio con selecciones de 25 cepas de Trichoderma entre ellas $T$. harzianum, de las cuales presentaron inhibición en $F$. oxysporum sobre el crecimiento de micelio en 33 y $35 \%$.

De igual manera, Sinuco et al. (2017) indican que los compuestos orgánicos volátiles de T. viride afectaron los halos de crecimiento de Fusarium spp. El crecimiento de T. asperellum mostró diferencias significativas $(p \leq 0.05)$ al confrontarse con los diferentes patógenos causantes de la marchitez del chile causados por los patógenos de $P$. capsici, $F$. oxysporum y $R$. solani $(p \leq 0.001)$ ya que al hacer el análisis de los datos se pudo observar que la tasa de crecimiento del patógeno se redujo prácticamente a la mitad al confrontarse con $T$. asperellum. 
Además, de que este antagonista sobrecreció sobre los patógenos hasta 100\%, cubriendo la caja completamente y causando así un hiperparasitismo, mostrando así una capacidad antagónica muy eficiente ante cada uno de los patógenos.

Estos resultados coinciden con otros autores quienes mencionaron que Trichoderma ssp. sobrecrece a Phytophthora causando un hiperparasitismo (Bae et al., 2016). De igual forma, Trichoderma spp. se desarrolla contra $R$. solani, causando la inhibición del crecimiento in vitro de $R$. solani de 58 a $86 \%$. Trichoderma spp., tiene la capacidad de inducir la expresión de genes relacionados con la defensa de las plantas y producir un mayor nivel de ergosterol, lo que indica su capacidad de crecer a un ritmo mayor en el suelo, esto explica sus efectos positivos sobre el crecimiento y la defensa de la planta en presencia del patógeno (Mayo et al., 2015).

Los aislamientos de Trichoderma confrontados con $P$. capsici ejercieron un efecto antagónico altamente significativo ( $p \leq 0.001$ ), causando la inhibición en el crecimiento del oomiceto en condiciones duales. T. harzianum, T. viride y T. asperellum, también tuvieron un comportamiento similar contra $F$. Oxysporum y $R$. solani (Figura 4). Los antagonistas continuaron creciendo hasta seis días después, mostrando hiperparasitismo al invadir o crecer completamente sobre el patógeno. Al respecto, Segarra et al. (2013), mencionaron que T. asperellum cepa T34, es capaz de reducir a $P$. capsici hasta $71 \%$, al aplicarse en plantas de pimiento en diferentes etapas de crecimiento.

\section{Micoparasitismo}

En los ensayos in vitro se evaluó la inhibición de las tres especies de Trichoderma, los resultados mostraron la eficacia en la competencia por espacio y nutrientes, además de apreciar la habilidad parasítica contra Rhizoctonia solani, Fusarium oxysporum y P. capsici. Además, s se observó mediante microscopía de luz y corroborar en los microcultivos y cultivos de doble confrontación en el punto de cruce entre los aislamientos nativos de Trichoderma spp. y patógenos causantes de la marchitez del chile los diferentes tipos de micoparasitismo (Figura 5).
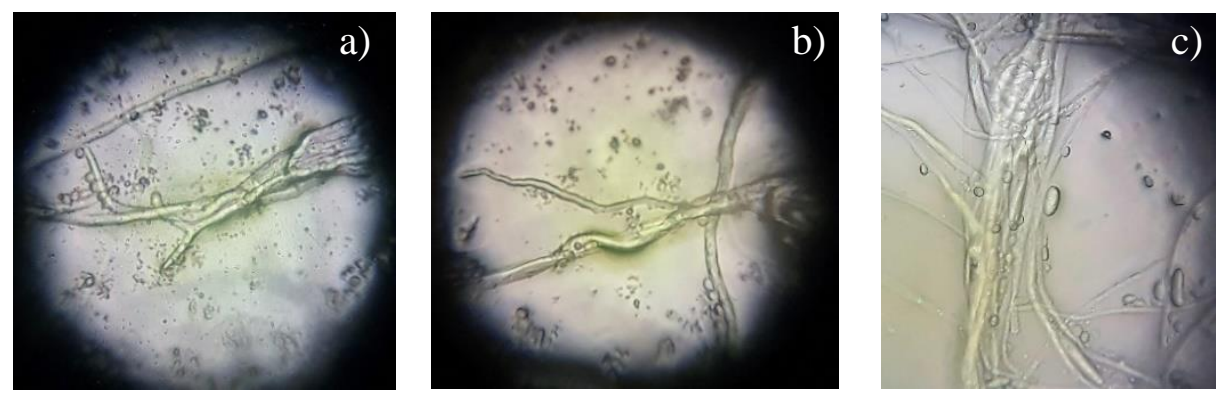

Figura 5. Micoparasitismo de Trichoderma sp. a) T. harzianum rodeando al micelio de Rhizoctonia solani; b) $T$. viride y $T$. asperellum rodeando el micelio de $P$. capsici; y c) enrollamiento masivo de T. asperellum cubriendo a Fusarium oxysporum.

En algunas primeras investigaciones se han observado mico-parasitismo, antibiosis, además de que Trichoderma tiene un comportamiento de crecimiento paralelo al patógeno hasta enrollarlo evitando los diferentes mecanismos de supervivencia del patógeno (Chet et al., 1981; Harman, 2006; Infante, 2009). 


\section{Conclusiones}

T. asperellum, $T$. viride y $T$. harzianum son antagónicos eficientes de los fitopatógenos que causan la marchitez del chile. Las pruebas con estos microorganismos antagonistas han demostrado la existencia de alternativas biológicas para el control de fitopatógenos de la raíz de chile en condiciones de laboratorio, por ello, se sugiere realizar evaluaciones de biocontrol con estos aislamientos y cultivo de chile en invernadero y a campo abierto. Esto coadyuvará para conocer el comportamiento en condiciones de competencia con una diversidad mayor de microorganismos y factores ambientales diferentes a los estudiados.

\section{Literatura citada}

Andrade, H. P.; De León, C.; Espíndola, B. M. C., Alvarado, R.D.; López, J. A. y García, E. R. 2012. Selección de porta-injertos de aguacate para tolerancia-resistencia a Phytophthora cinamomo Rands. Usando temperaturas controladas. Spanish J. Rural Development. 3(4):23-30.

Bae, S. J.; Mohanta, T. K.; Chung, J. Y.; Ryu, M.; Park, G.; Shim, S.; Hong, S. B.; Seo, H.; Bae, D. W.; Bae, I., Kim, J. J. and Bae, H. 2016. Trichoderma metabolites as biological control agents against Phytophthora pathogens. Biological Control. 92:128-138. Doi:10.1016/ j.biocontrol.2015.10.005.

Barnett, H. and Hunter, B. 1972. Illustrated genera of imperfect fungi. EE. UU. Burgess Publ. Co., 241p. Doi:10.2307/3757954.

Barnett, H. L. and Hunter, B. B. 1998. Illustrated genera of imperfect fungi. 4a. Ed. American Phytophatology Society, MN. 217 p.

Bouziane, Z.; Dehimet, L. and Kacem, C. N. 2016. Inhibitory activity of Trichoderma viride against Phytophthora infestans that affects the Spunta potato (Solanum tuberosum L.) variety. Afr. J. Microbiol. Res. 29(10):1121-1127.

Burgess, L. W.; Summerell, B. A.; Bullock, S.; Gott, K. P. and Backhouse, D. 1994. Laboratory manual for Fusarium research. $3^{\text {rd }}$ (Ed.). University of Sydney, Sydney, Australia. 132 p.

Chaverri, P.; Branco, R. F.; Jaklitsch, W.; Gazis, R.; Degenkolb, T. and Samuels, G. J. 2015. Systematics of the Trichoderma harzianum species complex and the re-identification of commercial biocontrol strains. Mycologia. 107(3):558-590.

Chet, I.; Harman, G. E. and Bake, R. 1981. Trichoderma hamatum: Its Hyphal Interactions with Rhizoctonia solani and Pythium spp. Microbial Ecol. 7(1):29-38.

de Hoog, G. S.; Guarro, J.; Gene, J. and Figueras, M. J. 2000. Atlas of clinical fungi, 2nd edition. entraalbureau voor Schimmelcultures, Utrecht. Mycological research. 106(4):507-511. Doi:10.1017/S0953756202226167.

Du Plessis, I. L.; Druzhinina, I. S.; Atanasova, L.; Yarden, O. and Jacobs, K. 2018. The diversity of Trichoderma species from soil in South Africa, with five new additions. Mycologia. 110(3):559-583.

Erwin, D. C. and Ribeiro, O. K. 1996. Phytophthora diseases worldwide. Department of Plant Pathology, University of California, Riverside, USA. American Phytopathol. Society. 562 p.

Ezziyyani, M.; Requena M. E.; Egea-Gilabert C. and Candela M. E. 2007. Biological Control of Phytophthora Root Rot of Pepper Using Trichoderma harzianum and Streptomyces rochei in Combination. Journal of Phytopathology. 155(6):342-349. Doi:10.1111/j.14390434.2007.01237.x. 
Ezziyyani, M.; Requena, M. A. y Candela, M. A. 2005. Producción de proteínas-PR en la inducción de resistencia a $P$. capsici en plantas de pimiento. Anales de Biología. 27:143-154.

Gallegly, M. E. and Hong, M. C. 2008. Phytophthora: identifying species by morphology and DNA fingerprints. American Phytopathol. Society. St. Paul, M. N. 158 p.

García, E. R. 2010. Agroecología y enfermedades de la raíz en cultivos agrícolas. Colegio de Postgraduados, estado de México, México. 130 p.

Granke, L. L.; Quezada, O. L.; Lamour, K. and Hausbeck, M. K. 2012. Advances in research on Phytophthora capsici on vegetable crops in the United States. Plant disease. 11(96):15881600.

Guédez, C.; Cañizalez, L. M.; Castillo, C. y Olivar, R. 2012. Evaluación in vitro de aislamientos de Trichoderma harzianum para el control de Rhizoctonia solani, Sclerotium rolfsii y Fusarium oxysporum en plantas de tomate. Rev. de la Sociedad Venezolana de Microbiología. 3(32):44-49.

Harman, G. E. 2006. Overview of Mechanisms and uses of Trichoderma spp. Phytopathology. 96(2):190-194. Doi: 10.1094/PHYTO-96-0190.

Harris, I. J. 1986. Modified method for fungal slide culture. J. Clinical Microbiol. 24(3):460-461.

Hermosa, R.; Viterbo, A.; Chet, I. and Monte, E. 2012. Plant-beneficial effects of Trichoderma and of its genes. Microbiology. 158(1):17-25. Doi: 10.1099/mic.0.052274-0.

Hernández, C. F. D.; Lira, S. R. H.; Gallegos, M. G.; Hernández, S. M. and Solis, G. S. 2014. Biocontrol of pepper wilt with three Bacillus species and its effect on growth and yield. Phyton. 83(1):49-55.

Hernández, H. E. J.; Hernández, R. I.; Almaraz, S. J. J.; López, L. A.; Torres, A. M. y Morales, F. F. J. 2018. Caracterización in vitro de rizobacterias y su antagonismo con hongos causantes del damping off en chile. Rev. Mex. Cienc. Agríc. 9(3):525-537.

Infante, D.; Martínez, B.; González, N. y Reyes, Y. 2009. Mecanismos de acción de Trichoderma frente a hongos fitopatógenos. Revista de Protección Vegetal. 1(24):14-21.

Jaklitsch, W.M. and Voglmayr, H. 2015. Biodiversity of Trichoderma (Hypocreaceae) in Southern Europe and Macaronesia. Studies in Mycology. 80:1-87. Doi.org/10.1016/j.simyco. 2014.11.001.

Jang, S.; Kwon, S. L.; Lee, H.; Jang, Y.; Park, M. S.; Lim, Y. W.; Kim C. and Kim, J. J. 2018. New report of three unrecorded species in Trichoderma harzianum species complex in Korea. Micology. 46(3):177-184.

Jhon, R. P.; Tyagi, R. D.; Prévost, D.; Brar, S. K.; Pouleur, S. and Surampalli, R. Y. 2010. Mycoparasitic Trichoderma viride as a biocontrol agent against Fusarium oxysporum $\mathrm{f}$. sp. adzuki and Pythium arrhenomanes and as a growth promoter of soybean. Crop Protection. 12(29):1452-1459.

Leandro, L. F. S.; Guzman, T.; Ferguson, L. M.; Fernandez G. E. and Louws F. J. 2007. Population dynamics of Trichoderma in fumigated and compostamended soil and on strawberry roots. Appl. Soil Ecol. 1(35):237-246.

Lozano, A. N.; Guzmán, P. R.; Zavaleta, M. E.; Aguilar, R. H.; Ayala, V. V. 2015. Etiology and evaluation of control alternatives for wilt in chile de árbol (Capsicum annuиm L.) in La Vega, Metzitlán, Hidalgo, México. 33(1):31-53.

Martínez, B.; Infante, D. y Reyes, R. Y. 2013. Trichoderma spp. y su función en el control de plagas en los cultivos. Revista de Protección Vegetal. 1(28):1-11.

Matar, S. M.; El-Kazzaz, S. A.; Wagih, E. E.; El-Diwan, A. I.; Moustafa, H. E.; Abo-Zaid, G. A.; Abd-Elsalam, H. E. and Hafez, E. E. 2009. Antagonistic and inhibitory effect of Bacillus subtilis against certain plant pathogenic fungi, I. Biotechnology. 8(1):53-61. 
Mayo, S.; Gutiérrez S.; Malmierca M. G.; Lorenzana A.; Campelo M. P.; Hermosa R. and Casquero P. A. 2015. Influence of Rhizoctonia solani and Trichoderma spp. In growth of bean (Phaseolus vulgaris L.) and in the induction of plant defense-related genes. Frontiers Plant Science. 6(685):1-11. Doi: 10.3389/fpls.2015.00685.

Molina, G. E.; Andrade, H. P.; García, E. R. y Sosa, H. C. M. 2016. Capacidad de sobrevivencia de tres especies de Phytophthora y dos de Pythium preservados en dos sustratos a corto y largo plazo. Rev. Mex. Cienc. Agríc. 7(7):1759-1764.

Nawaz, K.; Shahid, A. A.; Bengyell, L.; Subhani, M. N.; Alia, M.; Anwar, W.; Iftikhar, S. and Ali, S.W. 2018. Diversity of Trichoderma species in chili rhizosphere that promote vigor and antagonism against virulent Phytophthora capsici. Scientia Horticulturae. 239:242-252. Doi.org/10.1016/j.scienta.2018.05.048.

Osorio, H. E.; Hernández, C. F. D.; Gallegos, M. G.; Rodríguez, H. R. and Castillo, R. F. 2011. Invitro behavior of Trichoderma spp. Against Phytophthora capsici Leonian. Afr. J. Agric. Res. 19(6):4594-4600.

Osorio, H. E.; Hernández, F. D. C.; Rodríguez, R. H.; Varela, S. D. F.; Estrada, B. D. y López, J. A. S. 2016. Actividad antagónica de Trichoderma spp. sobre Rhizoctonia solani in vitro. Investigación y Ciencia. 67(24):5-11.

Osorio, H. E.; Hernández, M. J.; Conde, M. V.; Michel, A. A. C.; López, S. J. A. and Torres, C. J. A. 2016. In vitro activities of Trichoderma species against Phytophthora parasitica and Fusarium oxysporum. Afr. J. Microbiol. Res. 15(10):521-527.

Paul, B. 1999. Pythium periplocum, an aggressive mycoparasite of Botrytis cinerea causing the gray mould disease of grape-vine. FEMS Microbiology Lett. 181(2):277-80. Doi:10.1111/j.1574-6968.1999.tb08855.x.

Perveen, K. and Bokhari, N. A. 2012. Antagonistic activity of Trichoderma harzianum and Trichoderma viride isolated from soil of date palm field against Fusarium oxysporum. African J. Microbiol. Res. 13(6):3348-3353.

Rivera-Jiménez, M. N.; Zavaleta-Mancera, H. A.; Rebollar-Alviter, A.; Aguilar-Rincón, V. H.; García de los Santos, G.; Vaquera-Huerta, H.; Silva-Rojas, H. V. 2018. Phylogenetics and histology provide insight into damping-off infections of 'Poblano' pepper seedlings caused by Fusarium wilt in greenhouses. Mycological Progress. 17(11):1237-1249. Doi.org/10.1007/s11557-018-1441-2.

Romero-Arenas, O.; Amaro, J. L.; Damián, M. A.; Valencia de Ita, M. A.; Rivera, A. y Huerta, M. 2017. Biopreparados de Trichoderma spp. para el control biológico de Phytophthora capsici en el cultivo de tomate de Puebla, México. Información Técnica Económica Agraria.113(4):313-324. Doi.org/10.12706/itea.2017.019.

Romero, A. O.; Huerta, L. M.; Damián, H. M. A.; Domínguez, H. F. y Arellano, V. D. A. 2009. Características de Trichoderma harzianum, como agente limitante en el cultivo de hongos comestibles. Rev. Colomb. Biotecnol. 11(2):143-151.

Sabbagh, S. K.; Roudini, M. and Panjehkeh, N. 2017. Systemic resistance induced by Trichoderma harzianum and Glomus mossea on cucumber damping-off disease caused by Phytophthora melonis. Arch Phytopathol. Plant Protec. 50(5-8):375-3788.

Sánchez, G. B. M.; Espinosa, H. E.; Villordo, P. E.; Rodríguez, G. R. y Mora, A. M. A. 2017. Identificación molecular y evaluación antagónica in vitro de cepas nativas de Trichoderma spp. sobre hongos fitopatógenos de raíz en frijol (Phaseolus vulgaris 1.) cv. Montcalm. Agrociencia. 1(51):63-79.

SAS Institute Inc. 2002. SAS/STAT User guide. Release 9.0. North Carolina, USA. 1289. 
Segarra, G.; Avilés, M.; Casanova, E.; Borrero, C. and Trillas, I. 2013. Effectiveness of biological control of Phytophthora capsici in pepper by Trichoderma asperellum strain T-34. Phytopathol. Mediterránea. 1(52):77-83.

Seifert, K. 1996. Fusarium interactive key. Agric. Agri-Food. Canada. 65 p.

Singh, B. N.; Singh, A.; Singh, S. P. and Singh, H. B. 2011. Trichoderma harzianum-mediated reprogramming of oxidative stress response in root apoplast of sunflower enhances defence against Rhizoctonia solani. European Journal Plant Pathology. 1(131):121-134.

Sinuco, D. C.; Pérez, A. C.; Moreno, N. 2017. Evaluación de la actividad fungicida e identificación de compuestos orgánicos volátiles liberados por Trichoderma viride. Rev. Colomb. Biotecnol. 1(19):63-70.

Sneh B.; Burpee L. and Ogoshi A. 1991. Identification of Rhizoctonia species. American Phytopathology Society, MN. 133 p.

Sonnenbichler, J.; Lamm, V.; Gieren, A.; Holdenrieder, O, and Lotter, H. 1983. A Cyclopentabenzopyranone produced by the fungus Heterobasidion annosum in dual cultures. Phyrochemistry. 22(6):489-1491. Doi.org/10.1016/S0031-9422(00)84043-1.

Steel, R. G. D. y Torrie, J. H. 1986. Bioestadística: principios y procedimientos. 2a (Ed.). México: McGraw-Hill. 228 p.

Vásquez, L. A.; Tlapal, B. B.; Yáñez, M. M.; Pérez, R. P. y Quintos, M. E. 2009. Etiología de la marchitez del 'chile de agua' (Capsicum annuum L.) en Oaxaca, México. Rev. Fitotec. Mex. 2(32):127-134.

Verma, M.; Brar, S. K.; Tyagi, R. D.; Sahai, V.; Prévost, D.; Valéro, J. R. and Surapalli, R. Y. 2007. Bench-scale fermentation of Trichoderma viride on wastewater sludge: rheology, lityc enzymes and biocontrol activity. Enzyme and Microbial Technology. 41(6):764-771.

Woo, S. L. and Lorito, M. 2007. Exploiting the interactions between fungal antagonists, pathogens and the plant for biocontrol. In: ovel biotechnologies for biocontrol agent enhancement and management. Vurro, M; Gressel, J. N. (Eds.). Springer, Dordrecht. 107-130 pp.

Zegeye, D. E.; Santhanam A.; Gorfu, D.; Tessera, M. and Kassa, B. 2011. Biocontrol activity of Trichoderma viride and Pseudomonas fluorescens against Phytophthora infestans under greenhouse conditions. Inter. J. Agric. Technol. 6(7):1589-1602. 